\title{
Congenital contractural deformities of the fingers and arthropathy
}

J R MARTIN,${ }_{5}$ SHAO-NAN HUANG ${ }^{2}$ A LACSON,${ }^{2}$ R H PAYNE ${ }^{3} \mathrm{~S}$ BRIDGER F C FRASER, ${ }^{5}$ A J NEARY, ${ }^{6}$ E A MACLAUGHLIN, ${ }^{4}$ C HOBEIKA, AND क L J LAWTON

From the Disciplines of ${ }^{1}$ Medicine, ${ }^{2}$ Pathology, ${ }^{3}$ Basic Sciences, ${ }^{4}$ Radiology, ${ }^{5}$ Community Medicine, ${ }^{6}$ Surgery, and 'Paediatrics, Memorial University, St John's, Newfoundland, Canada

SUMMARY Four patients are described who presented with congenital finger contractures and arthropathy. There was synovial cell hyperplasia and giant cells but no inflammatory process: Radiographs showed flattening of the metacarpal and metatarsal heads and the proximal femorafl ossification centres. In the oldest patient the process had subsided leaving slight contractures bu甲 severe impairment of hip mobility. In another the arthropathy was still prominent in the early. teens. In a third, finger contractures had failed to respond to conservative or surgical measures.

Key words: camptodactyly, hypertrophic synovitis.

Congenital contractural deformities of the fingers and large joint arthropathy due to a noninflammatory hyperplasia of synovial tissue have been described by a number of observers. ${ }^{1-3} \mathrm{We}$ have studied two siblings and two unrelated cases with these features. In all the cases the parents were healthy, unrelated, Newfoundland Caucasians. There were no other individuals with contractures or relevant arthropathies in the three families. The purpose of this communication is to provide further observations on what appears to be a well defined syndrome.

\section{Case reports}

CASE 1

The youngest child of a family of six was the product of a normal full-term delivery. At birth her fingers were held in a clenched position; they could be passively extended but became progressively more fixed. Beginning at the age of 2 years a number of surgical attempts were made to correct the contractures in the thumbs, left ring, right index, middle, and little fingers. Hypertrophy of the sheaths, jelly-like fluid within the sheaths, and oedema or shortening, and atrophy of the tendons were re-

Accepted for publication 16 May 1985

Correspondence to Dr J R Martin, St Clare's Mercy Hospital, St John's, Newfoundland, Canada, A1C 5B8. corded on different occasions in the operatio descriptions. The tendon sheaths of one or mợ digits at a time were divided and restrictive bands removed, permitting full passive extension an\& tendon lengthening at two of the later operations The results were disappointing, and on each occa을 sion contractures returned and developed in the्ब hitherto unaffected little fingers. In early ado lescence the proximal interphalangeal (PIP) joints of the little fingers were arthrodesed.

During childhood she complained of pain in the elbows, knees, and ankles. One observer reporteç effusions but no heat or tenderness. At the age of 13 . years she was a well proportioned child with no. dysmorphic craniofacial features but with the finge deformities previously described. There was also कs slight flexion contracture of the left elbow and sligh? contractures of the PIP joints of the toes. The range of movement in the hips was normal apart from onl $10^{\circ}$ internal and external rotation. There were no effusions. Radiographs of the hands, skull, thoracia and lumbar spine, and pelvis were unremarkabløి apart from flattening of the metacarpal heads and an appearance suggestive of bilateral slipped femoraf epiphyses. The dermatoglyphic patterns did nof show the vertical alignment of the palmar lines seero in some other types of arthrogryposis. Other laboratory investigations, including haemogram, im munoglobulins, rheumatoid factor, serum comple 
ments urinary glycosaminoglycans, and amino acid levels, were normal or non-contributory. The fibroblast culture showed a full complement of 46XX normal chromosomes by Giemsa banding.

The tendon sheath excised at 2 years of age showed synoviocytic hyperplasia, focal stromal oedema, and surface fibrin deposition. At age 16 years the tendon sheath showed scarring. Ultrastructural examination of a tendon showed a few tendon fibrocytes, with cytoplasmic accumulation of loose filamentous strands and a mixture of densely and loosely packed fibrils. Focal disarray of collagen fibres was noted; these had a regular periodicity but were of varying diameters (50-175 nm). Amorphous electron dense finely granular material was abundant between collagen fibres, together with cellular fragments. The appearance of the skin, skeletal muscle, and fibroadipose tissue was unremarkable by both light and electron microscopy.

\section{CASE 2}

The oldest sibling of case 1 was a normal full-term baby. At birth it was noted that he did not straighten his fingers, though they could be extended passively but would return to a fixed position on release of pressure. Efforts to correct the deformity by physiotherapy and splinting were unsuccessful, and at $5 \frac{1}{2}$ years he was referred for surgical correction of the deformities. Swelling of the knees with patella taps and large wrists and ankles were noted. The flexor tendon sheaths were excised in digits of the left hand and a few weeks later in the thumb, index, and middle fingers of the right hand.

At the age of 23 years he began to complain of pains in the right hip which he ascribed to years of participation in contact sports. There were slight contractures of the elbows, PIP joints of the toes and the fingers, with lack of full flexion in the right ring finger. The left hip tended to rotate externally to $20^{\circ}$ when flexed, with only a few degrees of internal and external rotation from that position. Internal and external rotation were also slightly reduced in the right hip. The knees showed a somewhat boggy synovium but were otherwise unremarkable. The peronei were thin, the interossei wasted, and the knee tendon reflexes were brisk, but otherwise the physical examination was normal.

Laboratory investigations were normal. Radiographs were normal apart from extreme lordosis at L5-S1 and an appearance in the hands and pelvis similar to that of case 1 (Figs 1 and 2).

CASE 3

She was a normal full-term delivery, the second of three siblings, the other two being healthy boys. She was born with clenched fingers and later was found to have swelling of a number of joints. Splints were prescribed to straighten the fingers with some benefit. At 6 years of age the flexor pollicis tendon sheaths were released. During childhood she complained of intermittent pain in the left leg but participated in sports.

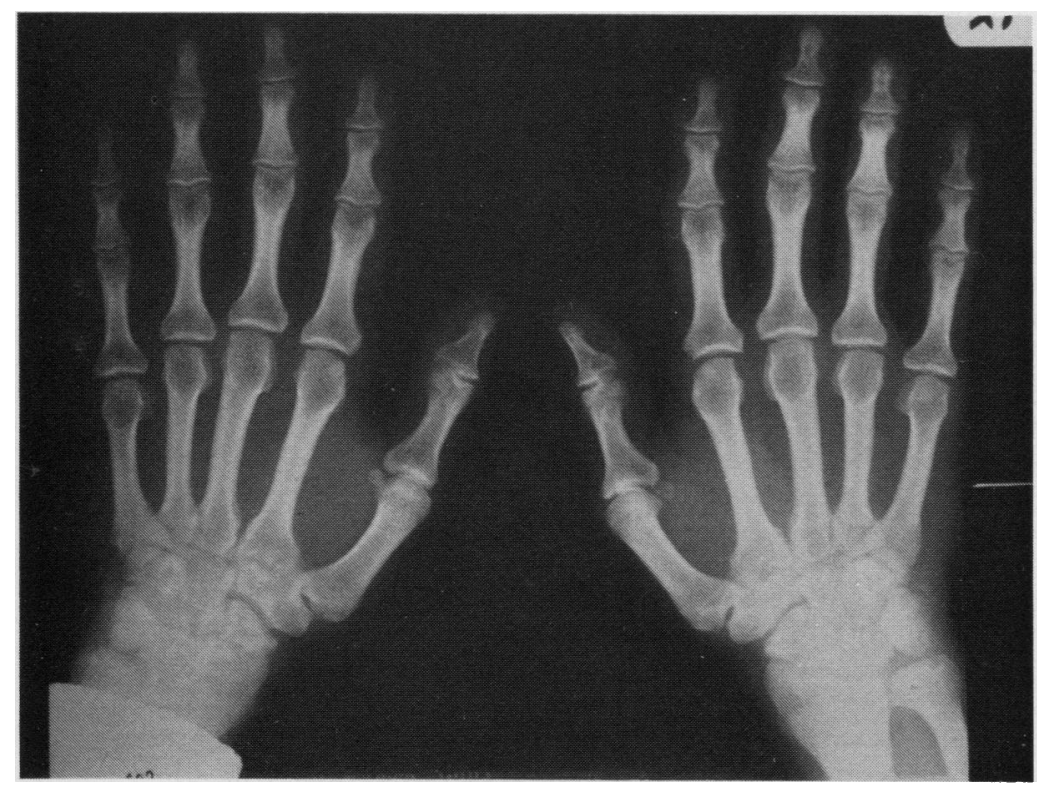

Fig. 1 AP view of hands. Flattening of the articular surface of metacarpal heads. Periarticular osteoporosis. Underdevelopment of styloid processes. 


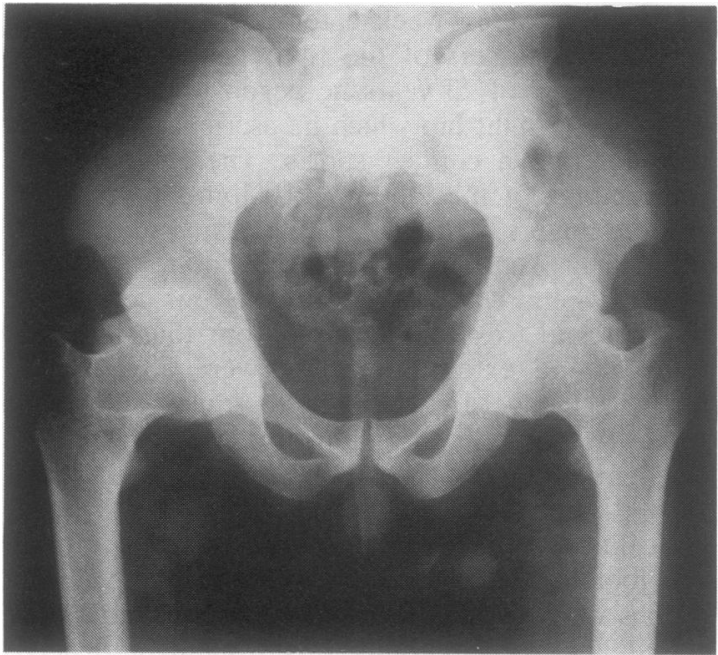

Fig. 2 AP view of pelvis at 24 years. Broadening and flattening of the proximal femoral epiphysis. The femoral necks are short and thick with a coxa vara deformity.

When seen at the age of 13 years she was a normally proportioned child with a steeple hard palate but no other dysmorphic features. Apart from a moderate myopia the abnormalities were restricted to the musculoskeletal system. They included swelling of the elbows, ankles, knees, several metacarpophalangeal (MCP) joints, and the sheaths of both extensor digitorum muscles. Contractures were present in the PIP joints, but the fingers could be fully clenched. There was marked impairment of movement in the hips and less in the ankles and wrists. Slight contractures were present in the toes, elbows, and knees and fixed $20^{\circ}$ contractures in both hips. There was a compensatory lordosis of the lumbar spine. Dermatoglyphic patterns were unremarkable.

Radiographs of the feet showed flattening of the metatarsal heads and an appearance of the pelvis and hands similar to that seen in case 2 . In the knees effusions were present and the femoral condyles were flattened.

Arthroscopic examination of the right knee showed grade I and II chondromalacia and extensive frond formation, which on histological examination showed extensive villus formation. Synoviocytic hyperplasia was evident, with oedema and focal collagen degeneration in the subsynovial zones (Fig. 3a). Many villi were coated with fibrin (Fig. 3b). Avascular, virtually acellular pink villi were present. Some villi were undergoing chondroid metaplasia. Human serum components - albumin, complements, and $\alpha_{1}$ antitrypsin were present in the cytoplasm of some synoviocytes (but not fibrinogen? and immunoglobulins A, G, and M) (Fig. 3c)? Multinucleated giant cells were easily seen among synoviocytes in both superficial and deep areas (Fig 3d). These had more or less central nuclei morphologically similar to those of adjacent synoviocytes $\overline{\bar{N}}$ The cytoplasm was abundant and homogenous showing a periodic acid-Schiff reaction and the same serum components as the synoviocytes.

CASE 4

Case 4 was a full-term delivery. There were twow miscarriages before the propositus and a normas male born subsequently. At the sixth week of $\bar{\alpha}$ pregnancy the mother had a slight bloody vaginat discharge and spent seven months in bed receiving medroxyprogesterone acetate every two weeks frombo six weeks to the eight months. At six months she began to have contractions and was then placed one Ventolin for the remaining part of the pregnancy.-

The child was born with all fingers clenched. At eight months she was found to have flexion deformities of the fingers at the PIP joints and recurvatums deformity of the MCP joints. She was prescribec night splints, and by 14 months the deformities had corrected themselves except for slight contractu民 and crepitus of the PIP joints of the ring and lit $\mathrm{e}^{\mathrm{er}}$ fingers. At 19 months puffiness, but no heat, of tec knees and ankles was noted, as well as swelling of the extensor tendons of the left wrist and a slight contracture of the right elbow. Hip movements wereڤ normal and there were no contractures of the toes $\underset{\mathscr{D}}{\mathbb{D}}$ She was placed on ASA $100 \mathrm{mg} / \mathrm{kg}$.

Pertinent laboratory findings: erythrocyte sedimentation rate $26 \mathrm{~mm} / 1 \mathrm{st} \mathrm{h}$ (Westergren), IgM-

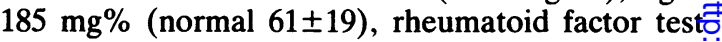
non-reactive. Radiographs showed an increased acetabular angle with abnormal indexes suggestive of a hip dysplasia.

\section{Discussion}

The four cases presented here have the typicalo features of the syndrome of congenital flexion contractures of the fingers and arthropathy. ${ }^{1-3}$ Theyo are similar to the type 1 distal arthrogryposis identified by Hall and her associates, ${ }^{4}$ though theo hands of the fourth case resemble their type II E. N The presence of the finger contractures at birth and N the absence of inflammatory cells in the synovium ${ }_{\sigma}^{\omega}$ distinguish them from the familial arthritis and camptodactyly reported by Malleson. ${ }^{5}$

The clinical course of the finger deformities wase quite variable. In cases 3 and 4 the finger deform:ities responded to conservative measures, and in case $e_{-}^{-}$ 2 simple incision of flexor tendon sheaths provided $\vec{D}$ 


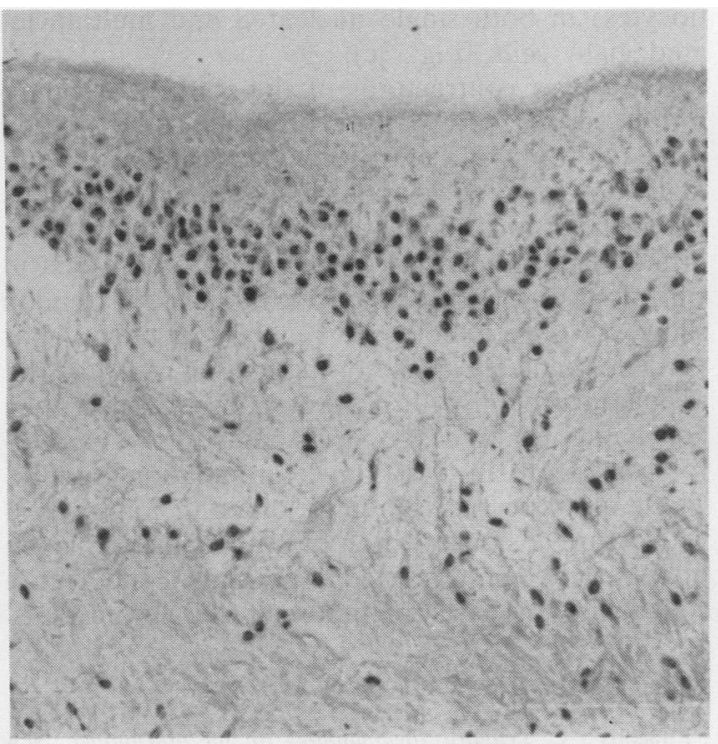

Fig. 3a

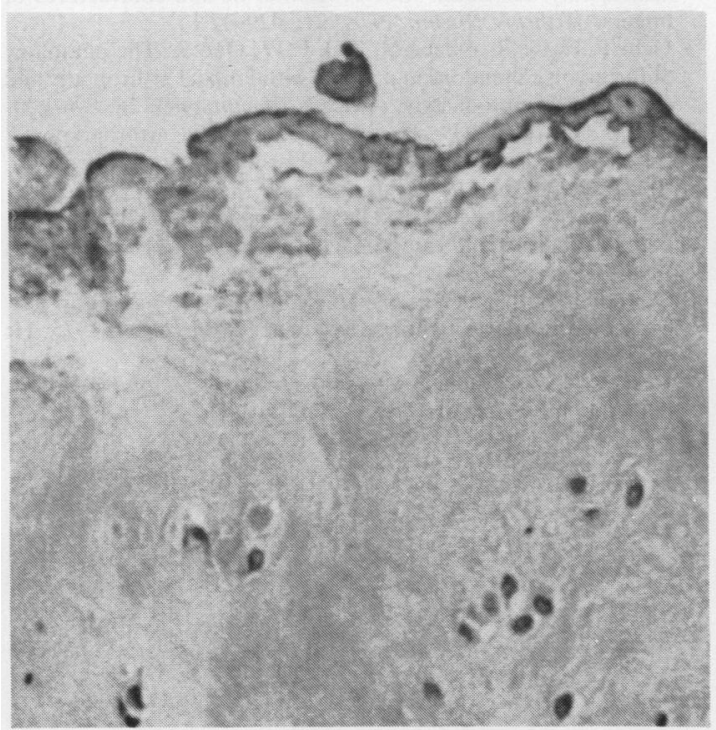

Fig. 3b

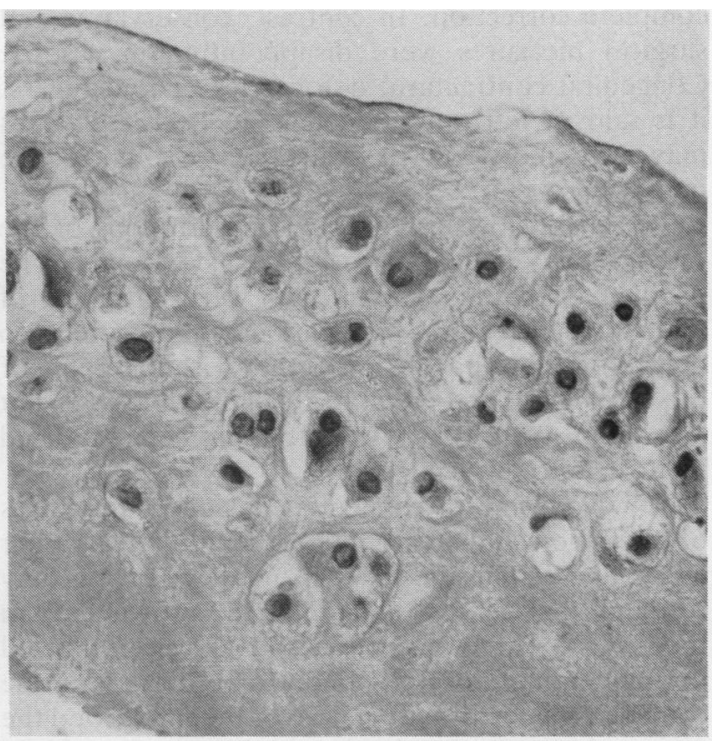

Fig. 3c

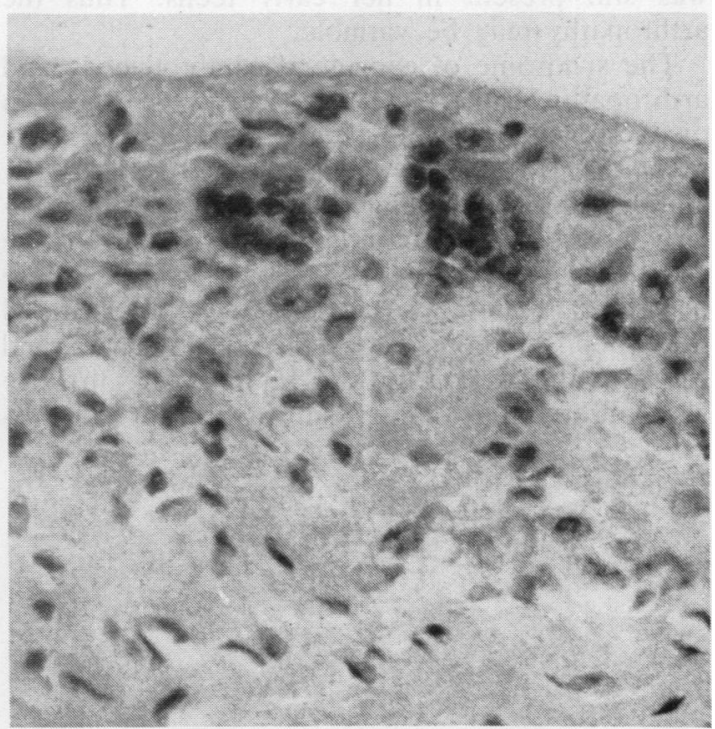

Fig. $3 d$

Fig. 3 (a) Villus showing hyperplasia of synoviocytes, with oedema and focal collagen degeneration in subsynovial zone. Inflammatory cells absent. (H and $E, \times 100)$. (b) Avascular villus with deposition of fibrin on surface and within collagenous tissues. (Immunoperoxidase staining for fibrin $\times 100$ ). (c) Avascular villus showing positive cytoplasmic staining of synoviocytes and chondrometaplasia. (Immunoperoxidase staining for $\alpha_{1}$ antitrypsin $\left.\times 200\right)$. (d) Several multinucleated giant cells in superficial and deep synovial zones. Note central uniform nuclei and homogenous cytoplasm. ( $H$ and $E, \times 200$ ). 
complete correction. In contrast, conservative and surgical measures were disappointing in case 1 . Congenital contractural deformities of the fingers, it is said, if left untreated tend to decrease ${ }^{2}$ but $^{2}$ may be improved or worsened by corrective operations. $^{36}$

There are no comprehensive descriptions of the radiographic appearance of this syndrome. The distinctive features in our cases were flattening of the proximal femoral ossification centres similar to that reported by Jacobs, ${ }^{1}$ together with flattening of the metacarpal and metatarsal heads, which was not present in Athreya's patients ${ }^{2}$ but has been reported in the Morquio syndrome. ${ }^{7}$

The syndrome of congenital flexion contractures of the fingers and arthropathy is said to affect larger joints and to subside early in infancy leaving only residual contractures and restricted movements in the hips. ${ }^{14}$ Three of our cases (1-3) were followed up long enough for an opinion to be formed on the course of the arthropathy. Two of them followed the expected course, but in the third (case 3 ) a florid polyarthritis affecting both large and small joints was still present in her early teens. Thus the arthropathy may be variable.

The syndrome of congenital camptodactyly and arthropathy begins in utero and affects the synovium of joints and tendon sheaths. ${ }^{1-3}$ The distinctive pathological features are synovial cell hypertrophy and hyperplasia, multinucleated giant cells, avascular villi, and absence of inflammatory cells. ${ }^{1-3}$ These changes were noted in those of our patients where histological material was examined. Athreya ${ }^{2}$ noted fibrin deposition on some of the villus surfaces, and we were able to confirm this finding (Fig. 3b). We also observed $\alpha_{1}$ antitrypsin (a marker for his- tiocytes) in both single nucleated and multinucle $\frac{3}{\infty}$
ated giant cells (Fig. 3c).

The finger contractures of the congenital camp $\Rightarrow$ todactyly and arthropathy syndrome are considered? to be the result of a tenosynovitis causing disruption of tendons and subsequent scarring and adhesions between tendons and tenosynovia. ${ }^{3}$ At various times different phases of this sequence were observed in our first case. Electronmicrographs of a tendorw showed collagen fibres of regular periodicity bu5 variable diameter. Some of the tendon cells had theappearance of myofibrocytes, suggesting that theyc are metabolically active and similar to the myofib-s roblasts seen in reparative granulation tissues These ultrastructural findings, taken in conjunction with Athreya's observation of intracellular collagen:fibres, could be interpreted as pointing to a defect in collagen synthesis as a component of the syndrome.N

\section{References}

1 Jacobs J C. Pediatric rheumatology for the practitioner. Ncu York: Springer-Verlag, 1982; 151-4.

2 Athreya B, Schumacker H R. Pathologic features of a familia arthropathy associated with congenital flexion contractures of
fingers. Arthritis Rheum 1978; 21: 429-37.

3 Ochi T, Iwase R, Okabe N, Fink C W, Ono K. The patholggy of the involved tendons in patients with familial arthropathy en congenital camptodactyly. Arthritis Rheum 1983; 26: 896-

4 Hall J G, Reed S D, Greene G. The distal arthrogrypos: Delineation of new entities - review and nosologic discussiono Am J Med Genet 1982; 11: 185-249.

5 Malleson P, Schaller J G, Dega F, Cassidy S B, Pagon R A Familial arthritis and camptodactyly. Arthritis Rheum 1981; 240 1199-204.

6 Goodman R, Gazit E, Kantznelson M B, et al. Four new heritable disorders of connective tissue. Birth Defects 1975; $11{ }^{7}$ 39-51.

7 Resnick D, Niwayama G. Diagnosis of bone and joint disorders Philadelphia: Saunders. 1981, 2599-602. 